\title{
Integrating patient perceptions within healthcare education: reflections and implications for pharmacy education and practice
}

Author: Beverley Lucas, Bradford School of Pharmacy, Bradford

Background: The importance of patient involvement as a positive contribution to healthcare education is now widely acknowledged. A literature review reported the potential to promote the learning of patient-centred practice, including inter-professional collaboration and community involvement. $^{1} \quad$ Further benefits suggested high learner satisfaction, recognition of the needs of vulnerable populations and increased confidence when learning clinical skills. An area for consideration arising from this review revealed the potential confusion around the language used to describe the spectrum of the patients' role, including the purpose of patient involvement and associated research strategies. A patient "spectrum of involvement" taxonomy ${ }^{1}$ was presented to address these difficulties. This matrix explores the degree to which the patient is actively involved in the learning encounter ranging from a focus on case based material to evidence of institutional commitment and sustained involvement.

Description of work: At this institution, the use of this patient "spectrum of involvement" taxonomy ${ }^{1}$ has informed our patient and public involvement strategy. Reflections on previous research related to an in-depth exploration of patient perceptions of their role in undergraduate medical education including benefits to students, patients and education ${ }^{2}$ within a primary care teaching practice ${ }^{3}$ will be offered.

Proposed evaluation: This presentation will also share experiences on the early application of the taxonomy within pharmacy in terms of educational practice and research and development opportunities. The first stage of data collection is focused on curricular mapping using the spectrum of involvement taxonomy to clarify the patient's role and facilitate communication of different initiatives. Drawing on suggestions for research foci ${ }^{1}$ four key areas will then be explored; drivers, structures, processes and outcomes of patient involvement.

\section{References}

1.Towle, A.,Bainbridge, L., Godolphin, W., Katz, A., Kline, C., Lown, B., Madularu, I., Solomon, P., Thistlethwaite, J. (2010). Active patient involvement in the education of health professionals, Medical Education; 44: (1), 64-74

2.Lucas, B., Pearson, D. (2012). Patient perceptions of their role in undergraduate medical education within a primary care teaching practice Education for Primary Care 23: $277-85$

3. Pearson, D., Lucas, B. (2011). Engagement and opportunity in clinical learning: Findings from a case study in primary care Medical Teacher; 33: e670-e677 